\title{
Intestinal bleeding from multiple small-bowel metastasis of an unknown primary melanoma - a case report
}

\author{
Enrico CB ${ }^{1 *}$, Angelo Alvino ${ }^{2}$, Adele Noviello ${ }^{1}$, Nicola Moles ${ }^{1}$, Antonio Miro ${ }^{1}$, Raffaele Iandoli ${ }^{3}$, Francesco Esposito ${ }^{1}$ and Francesco Crafa $^{1}$ \\ ${ }^{1}$ Department of Oncological and General Surgery, S.G. Moscati Hospital, Avellino, Italy \\ ${ }^{2}$ Departement of Emergency Surgery, S.G. Moscati Hospital, Avellino, Italy \\ ${ }^{3}$ Departement of Dermatology and Dermatosurgery, S.G. Moscati Hospital, Avellino, Italy
}

\begin{abstract}
We report here a case of 64-year-old man admitted to neurology department for a head injury with retrograde amnesia after a road accident. A CT-scan showed multiple bloody solid lesions of the small intestine and the patient underwent exploratory laparotomy. Histological examination found the presence of metastatic lesions from malignant melanoma. The physical dermatological examination of the patient allowed to identify an primary melanoma in the left scapular region. In the eighth edition of the American Joint Committee on Cancer (AJCC) melanoma staging system the site (s) of metastases and serum levels of LDH are used to delineate the M stage into four categories: M1a, M1b, M1c e M1d, each subcategorized in 0 (not elevated) and 1 (elevated) value of LDH. Actually, surgery have a important role in Stage IV disease for outcome and survival of the Disease.
\end{abstract}

\section{Introduction}

The American Cancer Society estimates that there are in 2017 in the United States 87,110 new skin melanoma diagnoses every year, and every year around 9,730 people die due to malignant melanoma [1]. The most important exogenous factor is exposure to UV irradiation, particularly intermittent sun exposure [2].

About $90 \%$ of melanomas are diagnosed as primary tumors without any evidence of metastasis. Melanomas can metastasize either by the lymphatic or the haematogenous route. About two-thirds of metastases are originally confined to the drainage area of regional lymph nodes. Distant metastases have a grim prognosis with a median survival in untreated patients being only 6-9 months, although there is considerable variation depending on internal organ involvement and serum levels of lactate dehydrogenase [3].

Intestinal metastases are more common in patients with advanced stage melanoma. Autopsy studies have shown that $60 \%$ of patients with malignant melanoma have secondary intestinal lesions, but only less than $5 \%$ of these are clinically diagnosed before death [4]. In patients with intestinal metastases from melanoma, the small bowel is the most affected area and are often asymptomatic. In this case the most frequently reported symptoms are: bleeding, abdominal pain, weight loss and bowel obstruction. Where possible, complete metastasectomy should always be performed [5].

The association of adjuvant chemotherapies in combination with new monoclonal antibodies could improve life expectancy in patients with intestinal or brain metastatic melanoma6. Nevertheless, the median survival for patients with metastatic melanoma ranges from 6.9 to 15.8 months.

\section{Case report}

A 64-year-old man was admitted to neurology department for a head injury with retrograde amnesia after a road accident. During the hospital stay, the patient presented an episode of melena and the biochemical texts confirmed the presence of anemia. A CT-scan showed multiple bloody solid lesions of the small intestine and the patient underwent exploratory laparotomy. Four solid extroflexed lesions in different location of small bowel were found. They had a size from 2 to $4 \mathrm{~cm}$ and a hard consistency (Figure 1).

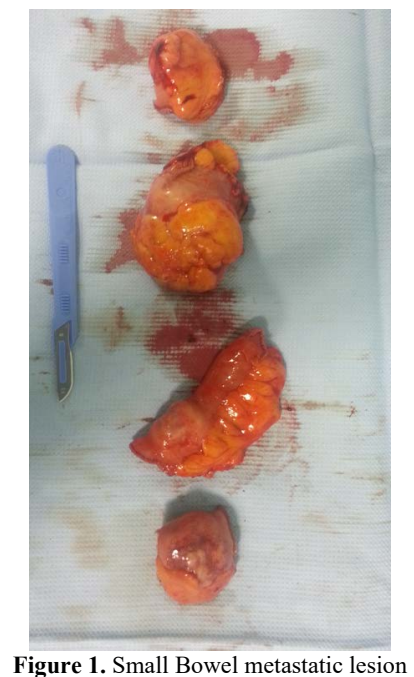

${ }^{*}$ Correspondence to: Enrico Coppola Bottazzi, Department of Oncological and General Surgery, S.G. Moscati Hospital, Avellino, Italy, Plesso Città Ospedaliera - Contrada Amoretta; 83100 Avellino, Italy, Tel: +393389981841; Email: enrico. coppolabottazzi@gmail.com

Key words: Intestinal metastases, malignant melanoma, stage IV, distant metastasis

Received:May 11, 2018; Accepted: May 22, 2018; Published: May 26, 2018 
Multiple segmental resections were performed with simultaneous anastomosis. The postoperative course was uneventful, and the patient was discharged to home on the fifth postoperative day. Histological examination found the presence of metastatic lesions from malignant melanoma with positive Ki67 70\%. The immunohistochemistry showed positivity for HMB45 and Melan A (MART1). Resected margins were free.

The physical dermatological examination of the patient allowed to identify an atypical pigmented lesion of about $11 \mathrm{~mm}$ in diameter with irregular edges in the left scapular region, which was surgically removed by a third level axillary lymphadenectomy for sentinel lymph node positivity (Figure 2). Histological examination revealed a malignant radial melanoma in regression with limited metastatic cutaneous infiltration and lymph node metastases.

The patient refused the adjuvant and follow-up. After a short time, he back to our ambulatory for a swell of the axillary region. He was subjected to biopsy confirming the presence of nodal metastasis. LDH level was $750 \mathrm{U} / \mathrm{l},(200-800 \mathrm{U} / \mathrm{l})$.

He died six months later from the later diagnosis.

\section{Discussion}

Small bowel is the second most common site of gastrointestinal metastasis following liver. Malignant melanomas of the small intestine can be primitive tumors or metastases of skin cancers and only $2 \%$ of melanomas appear as a primitive site to the small intestine.

Certain tumors frequently metastasizing in some organs and this is due to the expression of "homing signals" on tumor cells that direct their movement to appropriate microenvironments to produce metastatic lesions. In case of malignant melanoma, the CCR9 receptor expressed on tumor cells bind to its ligand CCL25 strongly expressed in the small bowel $[6,7]$.

In the eighth edition of the American Joint Committee on Cancer (AJCC) 8 melanoma staging system the site ( $\mathrm{s}$ ) of metastases and serum levels of $\mathrm{LDH}$ are used to delineate the $\mathrm{M}$ stage into four categories: M1a, M1b, M1c e M1d, each subcategorized in 0 (not elevated) and 1 (elevated) value of LDH. Patients with distant metastasis to skin, soft tissue including muscle, and/or non regional lymph node are categorized as M1a; patients with distant metastasis to lung with or without M1a sites of disease are categorized as M1b; Distant metastasis to non-CNS

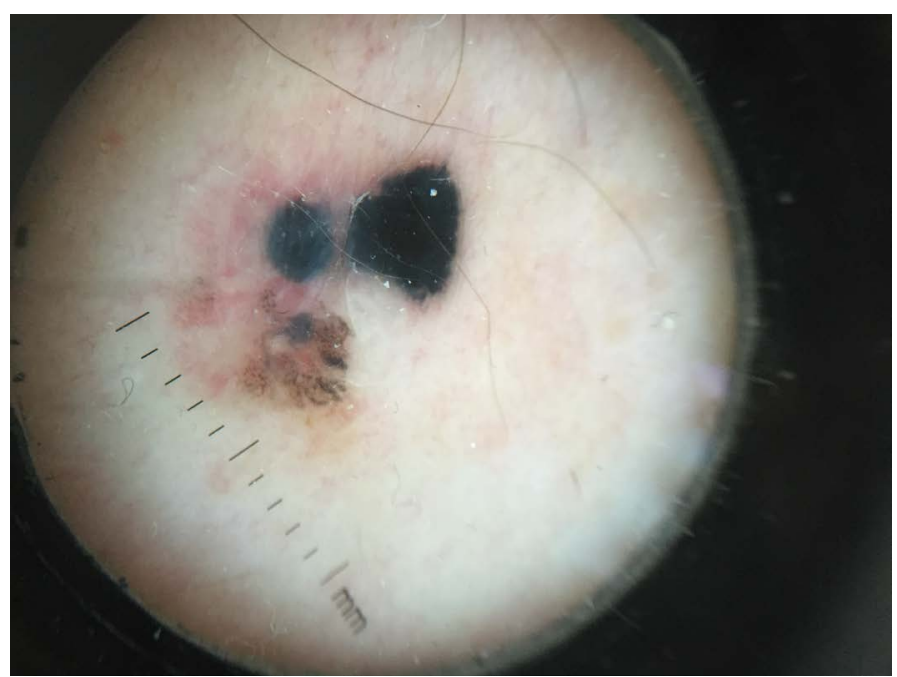

Figure 2. Primary melanoma of the left scapular region
(Central Nervous Sistem) visceral sites with or without M1a or M1b sites of disease are designated as M1c and M1d for distant metastasis to CNS with or without M1a, M1b, or M1c sites of disease. Elevated serum LDH are an independent and highly significant predictor of survival outcome. Infact LDH remains a clinically significant factor associated with response, progression-free survival, MSS (melanoma specific survival), and overall survival in the contemporary treatment era of targeted and immune therapies.

Faut and al, on a total of 22 patient affected by melanoma metastatized to the intestines, have developed a treatment algorithm of complication, especially bleeding and obstruction $[8,9]$. Generally, in patient with bleeding, in life treathering situation, a surgical urgency is necessary. In the case of oligometastatic melanoma disease in stage IV, limited to the intestine, with bleeding and life treathering situation a surgical resection is the treatment of choice.

If curative resection is not possible, a palliative resection of intestinal metastases can be an appropriate treatment option. It alleviates symptoms such as anemia, bleeding, abdominal pain, and/or weight loss in $90 \%$ of patients.

If symptoms such as bleeding are manageable, other options should be considered first. In patients with widely disseminated disease the possibilities of systemic treatment should first be explored for the treatment of intestinal metastases.

In case a melanoma harbors a BRAF mutation that can be targeted by a BRAF inhibitor, this is the treatment of first choice in patients with widely disseminated disease and high tumor load. The exceptionally fast and extensive responses that are regularly seen with these drugs are especially beneficial for patients with symptomatic and large metastases [10].

\section{Conclusion}

In the recent past, surgery had only a palliative role in stage IV melanoma, for example resecting small bowel metastases in case of bleeding or obstruction. Actually surgery have a important role in Stage IV disease for outcome and survival of the disease.

\section{Funding or grants}

None

\section{Conflict of interest}

None

\section{References}

1. American Cancer Society. Cancer Facts and Figures (2017) Atlanta, Ga: American Cancer Society Last accessed October 13.

2. Curtin JA, Fridlyand J, Kageshita T, Patel HN, Busam KJ, et al. (2005) Distinct sets of genetic alterations in melanoma. $N$ Engl J Med 353: 2135-2147. [Crossref]

3. Garbe C, Peris K, Hauschild A, Saiag P, Middleton M, et al. (2016) Diagnosis and treatment of melanoma. European consensus-based interdisciplinary guideline - Update 2016. Eur J Cancer 63: 201-217. [Crossref]

4. Lens M, Bataille V, Krivokapic Z (2009) Melanoma of the small intestine. Lancet Oncol 10: 516-521. [Crossref]

5. Ollila DW (2006) Complete metastasectomy in patients with stage IV metastatic melanoma. Lancet Oncol 7: 919-924. [Crossref]

6. Howard JH, Thompson JF, Mozzillo N, Nieweg OE, Hoekstra HJ et al. (2012) Metastasectomy for distant metastatic melanoma: analysis of data from the first Multicenter Selective Lymphadenectomy Trial (MSLT-I). Ann Surg Oncol 19: 25472555. [Crossref] 
7. Richmond A (2008) CCR9 homes metastatic melanoma cells to the small bowel. Clin Cancer Res 14: 621-623. [Crossref]

8. Gershenwald JE, Scolyer RA, Hess KR, Sondak VK, Long GV, et al. (2017) Melanoma staging: Evidence-based changes in the American Joint Committee on Cancer eighth edition cancer staging manual. CA Cancer J Clin 67: 472-492. [Crossref]
9. Faut M, Bisschop K, Jalving M, Been LB, Noordzij W, et al. (2017) Retracted: Diagnosis and Treatment of Intestinal Melanoma Metastases in the Era of Effective Systemic Treatment. Ann Surg Aug 22. [Crossref]

10. Hauschild A, Grob JJ, Demidov LV, Jouary T, Gutzmer R, et al. (2012) Dabrafenib in BRAF-mutated metastatic melanoma: a multicentre, open-label, phase 3 randomised controlled trial. Lancet 380: 358-363. [Crossref]

Copyright: $\odot 2018$ Enrico CB. This is an open-access article distributed under the terms of the Creative Commons Attribution License, which permits unrestricted use, distribution, and reproduction in any medium, provided the original author and source are credited. 